\title{
El uso de un recurso digital para la creación de historietas a partir de estrategias de planificación multinivel
}

\section{Sandra Paola Villagrán Olivares}

Licenciada en Educación Especial y auxiliar de primera de la asignatura Educación Especial del Profesorado de Educación Especial de la Facultad de Ciencias Humanas de la Universidad Nacional de San Luis spvo36@gmail.com

\begin{abstract}
María Fernanda Pahud Morales
Licenciada en Gestión de la Educación Especial, especialista en Educación Superior, profesora asociada de la Licenciatura en Educación Especial de la Facultad de Ciencias Humanas de la Universidad Nacional de San Luis y directora de Línea de Investigación

fernandapahud@gmail.com

\section{Mariela Zuñiga Velázquez}

Profesora en Ciencias de la Computación, especialista en Educación Superior y docente responsable de Práctica del Departamento de Informática de la Facultad de Ciencias Físico, Matemáticas y Naturales de la Universidad Nacional de San Luis marielagood@gmail.com
\end{abstract}

\section{Extracto}

Este trabajo tiene la intención de plantear la experiencia realizada con una herramienta digital para generar cómics en el marco de la asignatura Tecnologías Aplicadas a la Educación Inclusiva del segundo año del ciclo de complementación curricular Licenciatura en Educación Especial de la Facultad de Ciencias Humanas de la Universidad Nacional de San Luis (Argentina).

La experiencia consistió en articular propuestas para favorecer el enriquecimiento del lenguaje tecnológico de los futuros licenciados, con la planificación multinivel como estrategia de programación curricular inclusiva. Para esto se recurrió a una herramienta digital como Playcomic y a las posibilidades que esta herramienta ofrece para realizar programaciones de actividades educativas inclusivas.

Las lecturas a partir del análisis de la experiencia realizada con este grupo de estudiantes resultaron interesantes, desafiantes y productivas. Nos encontramos frente a un cambio de escenario que requiere, por parte de todo el sistema educativo, una fuerte y continua capacidad de autorreflexión. Los actuales lineamientos legales fomentan el desarrollo de procesos inclusivos a nivel educativo, exigiendo que el sistema se adecue para atender a la diversidad en su amplio espectro y no haciendo un reduccionismo simplista pensando que la diversidad solo tiene que ver con las personas con discapacidad.

Palabras clave: lenguaje tecnológico; planificación multinivel; herramienta digital; educación inclusiva; prácticas docentes.

Fecha de entrada: 03-07-2019 / Fecha de revisión: 22-07-2019 / Fecha de aceptación: 28-08-2019

Cómo citar: Villagrán Olivares, S. P., Pahud Morales, M. ${ }^{a}$ F. y Zuñiga Velázquez, M. (2020). El uso de un recurso digital para la creación de historietas a partir de estrategias de planificación multinivel. Tecnología, Ciencia y Educación, 15, 165-176. 


\title{
The use of a digital resource for the creation of cartoons from multilevel lesson planning strategies
}

\author{
Sandra Paola Villagrán Olivares \\ María Fernanda Pahud Morales \\ Mariela Zuñiga Velázquez
}

\begin{abstract}
This presentation has the main intention of proposing the experience made with a tool which was used in order to generate comics in the frame of Technologies Applied to an Inclusive Education from the second year of the complementary cycle of the bachelor's degree in Special Education, San Luis National University.

The experience consisted in articulating proposals to favor the enrichment of the technological language of future graduates, with multilevel planning as an inclusive curricular programming strategy. Using a digital tool like Playcomic and the possibilities that this tool offers to carry out programming of inclusive educational activities.

The readings from the analysis of the experience carried out with this group of students were interesting, challenging and productive. We are facing a scenery change that requires a strong and continuous capacity for self-reflection on the part of the entire educational system. The current legal guidelines encourage the development of inclusive processes at the educational level, demanding that the system be adapted to address diversity in its broad aproach and not making a simplistic reductionism thinking that diversity only has to do with people with disabilities.
\end{abstract}

Keywords: technological language; multilevel lesson planning; digital tool; inclusive education; teaching practices.

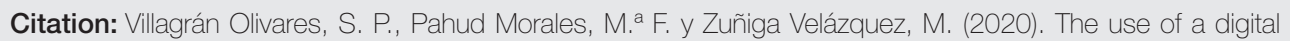
resource for the creation of cartoons from multilevel lesson planning strategies. Tecnología, Ciencia y Educación, 15, 165-176. 


\section{Sumario}

1. Introducción

2. Metodología de trabajo de la que partimos

3. Acerca de los participantes de la experiencia

4. Acerca de la asignatura Tecnologías Aplicadas a la Educación Inclusiva

5. Presentación de la herramienta digital Playcomic

6. Marco teórico acerca de la planificación multinivel como estrategia inclusiva y la herramienta digital Playcomic

7. Acerca de la propuesta práctica del recurso digital

8. Conclusiones acerca de la experiencia

9. Consideraciones finales

Referencias bibliográficas 


\section{Introducción}

El uso y la apropiación de recursos digitales inclusivos que enriquezcan las diversas propuestas que se desarrollan en las prácticas docentes, como, por ejemplo, la posibilidad de planificar clases que incluyan a todo el alumnado, resulta un gran desafío. En este sentido es muy importante comprender, tal como propugnamos desde nuestra línea de investigación ${ }^{1}$, que el lenguaje general, en el sentido vigotskiano, es un instrumento que regula el pensamiento y la acción (Zuñiga y Pahud, 2016), siendo el «lenguaje tecnológico», en particular, un instrumento de comunicación y de acción que contribuye con el desarrollo de nuevas capacidades de pensamiento que fomentan la creación de prácticas docentes inclusivas. Desde este marco referencial, el uso de recursos digitales cobra un sentido muy relevante, sobre todo, centrándonos en los postulados de Gloria Eldestein (2011) cuando manifiesta que formadores de forma-

El uso de recursos digitales cobra un sentido muy relevante, sobre todo, centrándonos en los postulados de Gloria Eldestein cuando manifiesta que formadores de formadores usan diferentes recursos tecnológicos para favorecer la comprensión de diversas temáticas en la convicción de que «la forma también es contenido»
El lenguaje general, en el sentido vigotskiano, es un instrumento que regula el pensamiento y la acción, siendo el «lenguaje tecnológico», en particular, un instrumento de comunicación y de acción que contribuye con el desarrollo de nuevas capacidades de pensamiento que fomentan la creación de prácticas docentes inclusivas dores usan diferentes recursos tecnológicos para favorecer la comprensión de diversas temáticas en la convicción de que «la forma también es contenido" (citado en Pahud y Zuñiga, 2016, p. 139). En este aspecto, la experiencia llevada a cabo con el grupo de futuros licenciados hizo sinergia en un doble sentido. Por un lado, proporcionándoles formación en un área del conocimiento muy compleja y, que suele generar resistencias,

1 Línea 2: Prácticas de Enseñanza. Lenguaje Tecnológico y Comprensión en Espacios de Formación en la Carrera del Profesorado de Educación Especial, dirigido por la especialista María Fernanda Pahud, perteneciente al PROICO núm. 4-1316 (Prácticas de Enseñanza para la Comprensión. Su Impacto en la Formación de los Estudiantes de las Carreras de Profesorados de Educación Inicial y Especial) de la Facultad de Ciencias Humanas de la Universidad Nacional de San Luis, dirigido por la especialista Zulma Escudero. 
como lo es el uso de la tecnología en entornos educativos. Y, por otro lado, ofreciéndoles herramientas pedagógicas para impactar en los procesos de enseñanza en los diferentes escenarios educativos en los que trabajan.

\section{Metodología de trabajo de la que partimos}

En un primer momento, la propuesta de la asignatura consistió en el abordaje de conceptos teóricos nucleares para el desarrollo de la materia. Esta primera etapa permitió la reflexión respecto a lo que venimos analizando en nuestra línea de investigación, referido a cómo el lenguaje tecnológico no solo posee una faz instrumental, sino que va más allá, ya que «implica un uso crítico del mismo por parte de los sujetos, que los posicione en un rol activo, permitiéndoles, a través del desarrollo de nuevas herramientas y capacidades de pensamiento, transformar su entorno» (Pahud y Zuñiga, 2018, p. 2).

En un segundo momento, la propuesta consistió en presentar herramientas digitales que ofrecen la posibilidad de desarrollar el uso crítico de la tecnología para lograr la evaluación, el diseño y la implementación de recursos didácticos digitales con las características propias de entornos educativos inclusivos. Dentro de este encuadre, se solicitó a los estudiantes la elaboración del trabajo final, que consistió en el diseño de un recurso didáctico digital utilizando alguna de las aplicaciones presentadas.

\section{Acerca de los participantes de la experiencia}

Los alumnos de la asignatura son egresados del Profesorado de Educación Especial y, algunos de ellos, del Profesorado de Enseñanza Diferenciada que cursan el Ciclo Complementario de Licenciatura en Educación Especial. La mayoría desempeña el rol de maestro de apoyo a la integración tanto en escuelas estatales como privadas, así como también en centros educativos terapéuticos. Otro gran porcentaje de estudiantes trabaja en consultorios particulares, desarrollando actividades de apoyo escolar y de acompañamiento a los procesos de inclusión educativa.

La mayoría de los estudiantes manifestó poseer un nivel bajo de competencias digitales para poder aplicarlas a sus propias prácticas docentes. En lo que respecta al uso de la tecnología dentro del ámbito personal, se manifestaron más proactivos y seguros en la utilización de herramientas tales como YouTube, Drive, WhatsApp, Google, Wikipedia, correo electrónico, etc., pero, en menor medida, con el uso de blogs, de Google Académico, Padlet, Moodle, Skype, etc. También manifestaron poseer buena conectividad en sus lugares de trabajo y contar con salas de computación, proyectores digitales, acceso a impresoras, tablets, netbooks y notebooks. 


\section{Acerca de la asignatura Tecnologías Aplicadas a la Educación Inclusiva}

La materia consta de un crédito de 6 horas semanales de corte teórico-práctico, de las cuales 3 horas son presenciales y 3 horas son no presenciales, en las que se plantean propuestas para desarrollar desde el aula virtual, entendida esta como aula extendida.

La asignatura se organizó en torno a tres unidades didácticas destinadas a colaborar en la formación de los estudiantes con el objetivo de generar estrategias necesarias que permitan procesos tales como la identificación, el análisis y la selección de diversos recursos tecnológicos disponibles que sean factibles de ser transferidos a otros entornos educativos. De un modo general, las dos primeras unidades versaron sobre temas vinculados a la tecnología de la información y la comunicación (TIC) y a su implementación en los ámbitos educativos como enriquecedores de propuestas pedagógicas innovadoras y como herramientas para organizar la información. A su vez, de manera transversal, se propuso el abordaje de conceptos que vienen articulados con el modelo social de la discapacidad, desde donde entendemos las prácticas inclusivas: la accesibilidad universal, las tecnologías de apoyo, el diseño universal de aprendizaje, la lectura fácil, la planificación multinivel, entre otros.

En la unidad 3, denominada «La tecnología digital para la producción y evaluación de recursos didácticos», se trabajaron algunos recursos digitales (Canva -para crear infografías-, Cuadernia, Videopad, distintas aplicaciones móviles, etc.) con la intención de desarrollar en los estudiantes la posibilidad de experimentar, analizar y evaluar los posibles entornos de aplicación didáctica de los mismos. En este artículo, recuperamos el trabajo que hicimos con el recurso digital Playcomic y su vinculación con la planificación multinivel.

\section{Presentación de la herramienta digital Playcomic ${ }^{2}$}

Los criterios de selección del recurso Playcomic se basaron en las posibilidades que ofrece la herramienta digital para incorporar un tipo de planificación didáctica favorecedora de prácticas educativas inclusivas, como lo es la planificación multinivel. Los objetivos para la formación del licenciado en Educación Especial versaron en generar un espacio para conocer las posibilidades peda-

\begin{abstract}
Los criterios de selección del recurso Playcomic se basaron en las posibilidades que ofrece la herramienta digital para incorporar un tipo de planificación didáctica favorecedora de prácticas educativas inclusivas, como lo es la planificación multinivel
\end{abstract}

2 La dirección de acceso a la herramienta es <http://ntic.educacion.es/w3/eos/MaterialesEducativos/mem 2009/playcomic/spanish/accesible/instrucciones_a.html>. 
gógicas que ofrece Playcomic y la implementación de secuencias didácticas en función de la planificación multinivel.

Playcomic es una herramienta online gratuita que se muestra como una opción interesante para trabajar con estudiantes que tienen discapacidad intelectual y discapacidad sensorial auditiva y que presentan diferentes niveles de competencia curricular en las diversas áreas del conocimiento, en especial en aquellas vinculadas con el desarrollo del lenguaje. El recurso digital está diseñado para que los estudiantes puedan elaborar, modificar y crear fácilmente historietas de una manera sencilla y atractiva. La herramienta ofrece múltiples opciones que permiten diseñar actividades para favorecer la agilización de los procesos de pensamiento (por ejemplo, trasladar pensamientos e ideas a las estructuras formales que ofrece el lenguaje escrito) y fomenta la posibilidad de traducir las informaciones icónicas a verbales, y viceversa.

A su vez, esta aplicación es una herramienta versátil, que ofrece distintos niveles de complejidad para crear historietas en castellano y en inglés: modificar un cómic ya existente, convertir una historia escrita en viñetas o inventar una nueva historieta desde el principio. Los estudiantes o usuarios pueden escoger entre más de 10 personajes, cambiar su posición y tamaño, y añadirles efectos sencillos, como líneas de movimiento y otros.

El recurso cuenta también con onomatopeyas, posibilidad de selección de escenarios o fondos, globos de diálogo básicos, textos de tipo artístico que se pueden seleccionar, pancartas o carteles, efectos variados, etc. El programa permite modificar algunas expresiones faciales básicas y cuenta con herramientas internas para el uso del software que son fáciles de comprender y de utilizar, ya que responden a un tipo de lectura fácil derivada del diseño universal de aprendizaje. Permite guardar los trabajados realizados para seguir modificándolos o posteriormente imprimirlos.

Las limitaciones, las desventajas o los puntos frágiles de la herramienta son pocos; sin embargo, se debe tener en cuenta que, por ejemplo, las letras de los globos, en una primera visualización, resultan muy pequeñas, pero luego se pueden ir eligiendo diferentes tamaños; lo mismo sucede con algunas de las viñetas e imágenes.

En lo que respecta a los requisitos técnicos, se puede trabajar en ordenadores con sistema operativo Windows. El lenguaje de programación utilizado en su creación ha sido Action Script 2.0 para Flash. Se puede trabajar con el software en diferentes resoluciones de pantalla, pero no puede ser utilizado en tablets comunes, ya que el lenguaje utilizado para su confección lo imposibilita. 
En cuanto a las características generales de esta aplicación y sus posibilidades didácticas, destacamos que se trata de un recurso gratuito que permite el diseño, la edición y la expresión escrita de los estudiantes con una adecuada escala de complejidad, según las posibilidades de los grupos con los que se trabaja. Es una herramienta motivadora por sus componentes lúdicos, siendo importante ponderar como muy positivo que el recurso ofrece una versión accesible. También cuenta con una guía rápida y otra detallada sobre las posibilidades que ofrece el software, que es de gran ayuda para los docentes. Si bien se trata de un recurso digital muy accesible, es recomendable para niños desde los 10 años en adelante, así como para jóvenes y adultos con discapacidad intelectual o sensorial auditiva con un adecuado desarrollo de la motricidad fina, ya que el recurso requiere el uso del ratón o del panel táctil.

Asimismo, por las características antes mencionadas, consideramos que Playcomic es un recurso muy interesante y potente como herramienta de apoyo para el proceso de enseñanza y aprendizaje de la lectura y de la escritura en personas con discapacidad intelectual, con discapacidad sensorial auditiva y con otras dificultades específicas de aprendizaje.

Playcomic es una herramienta que se adecua a distintos estilos de aprendizaje, pero, en especial, es muy útil para aquellos estudiantes que manifiesten una mayor inclinación por el trabajo con imágenes, a los que les guste la narrativa en sus variadas formas.

\section{Marco teórico acerca de la planificación multinivel como estrategia inclusiva y la herramienta digital Playcomic}

La planificación multinivel consiste en un conjunto de adaptaciones y estrategias que se adoptan en el aula para dar respuesta a los diferentes niveles de los grupos, disminuyendo la necesidad de tener programas diferenciados, generando prácticas educativas que incluyan a todos los estudiantes, sin necesidad de usar planificaciones diferentes o descontextualizadas del conocimiento que está circulando en un aula. La planificación multinivel permite introducir objetivos individuales en los contenidos y en las estrategias educativas previstas para el trabajo de aula, contemplando a todos los estudiantes, generando instancias de aprendizaje que ofrezcan la confluencia simultánea de distintas tareas y ritmos de trabajo en un mismo momento pedagógico. Es una estrategia que posibilita entornos de aprendizaje colaborativos, diversificados, accesibles y con variados canales de acceso al conocimiento (Villagrán, 2018).

\section{La planificación multinivel consiste en un conjunto de adaptaciones y estrategias que se adoptan en el aula para dar respuesta a los diferentes niveles de los grupos, disminuyendo la necesidad de tener programas diferenciados, generando prácticas educativas que incluyan a todos los estudiantes, sin necesidad de usar planificaciones diferentes o descontextualizadas del conocimiento que está circulando en un aula}


El recurso digital Playcomic permite la producción de historietas de forma escalada, es decir, con varios niveles de dificultad. Precisamente, está pensado para hacer más accesible el recurso a todos los alumnos, tanto a los que están alfabetizados como a aquellos otros que aún están en proceso de alfabetización. Estas características del recurso fueron muy importantes para la vinculación con la planificación multinivel, ya que la gradación de las actividades va permitiendo al docente analizar los niveles de intervención que irá proporcionando (de mayor a menor), fomentando, de este modo, la autonomía del estudiante y un mejor seguimiento y valoración de las actividades de enseñanza-aprendizaje, con criterios bien explícitos para todos los involucrados en dicho proceso.

Cuando se planifican actividades educativas desde la perspectiva multinivel, la evaluación de dichas actividades también debe ser considerada desde esta perspectiva. Para ello es necesario desglosar niveles de evaluación que no se alejen del objetivo general, pero que contemplen diferentes niveles de complejidad que se puedan ir ponderando, según las competencias de los estudiantes, en congruencia con las actividades que se van definiendo. A nivel más relacional, esta forma de planificación de las situaciones educativas ofrece entornos de aprendizaje basados en la aceptación, la seguridad y la confianza mutua entre los docentes y los estudiantes.

\section{Acerca de la propuesta práctica del recurso digital}

Dentro de la propuesta de la asignatura, se ofrecieron dos clases para abordar la complejidad de la tarea propuesta. Una de las clases se destinó a la presentación del recurso digital, sus potencialidades y debilidades y sus vínculos con prácticas educativas inclusivas, como la planificación multinivel, abordando en profundidad este tipo de planificación. La segunda clase se destinó a la presentación de los recursos digitales que los mismos estudiantes realizaron, fundamentando sus elecciones ante el grupo total de compañeros. Entre ambos encuentros presenciales (clases), se ofrecieron distintos espacios de consulta, tanto en el aula virtual como de forma presencial, los cuales resultaron muy productivos.

La mayor dificultad manifestada por los estudiantes estuvo en la comprensión y posterior aplicación de una secuencia didáctica basada en la planificación multinivel. Esta situación nos llevó a la reflexión, en tanto se trataba de un grupo de estudiantes ya con formaciones previas y con mucha experiencia en temas vinculados con programaciones didácticas en el marco de sus propias prácticas docentes. Sin embargo, a través de los espacios de consulta, se pudieron ir despejando las dudas y mostrando a los estudiantes que este tipo de planificación es un aliado muy importante para poder generar buenas prácticas docentes, ancladas en una visión de la educación que resulta genuinamente inclusiva.

Para las secuencias didácticas, se sugirió que se diseñaran objetivos específicos para, al menos, dos niveles de competencia curricular. Las secuencias didácticas debían contener 
aspectos mínimos, tales como inicio, introducción, actividades de cierre o evaluación. Los estudiantes podían elegir entre las variadas opciones que ofrece el recurso Playcomic y adaptar dichas propuestas al grupo de alumnos al cual fuera destinado.

La propuesta práctica o de aplicación consistió en el diseño de una secuencia didáctica en función de la planificación multinivel. En el caso de los estudiantes que estaban trabajando bajo la modalidad de maestro de Apoyo a la Integración, la tarea consistió en la realización de una planificación en función de dos niveles de competencia curricular, el del alumno con el que estaban trabajando y el del grupo total del aula en la cual realizaban la integración. Para ello podían estimar el nivel de competencia curricular y otros aspectos necesarios para desarrollar la planificación, sin necesidad de realizar diagnósticos pedagógicos individuales.

A modo de sugerencias orientativas para las tareas que se debían desarrollar, se propuso realizar una caracterización precisa del grupo de alumnos, describiendo estilos de aprendizaje, competencias curriculares, potencialidades y limitaciones frente al aprendizaje. En este punto se les solicitó que hicieran especial énfasis en los desempeños curriculares de los estudiantes, identificándolos en los dos niveles de competencia curricular que abordarían luego en las propuestas. Se les pidió que determinaran un área para trabajar que estuviese vinculada con el lenguaje, con las actividades de la vida diaria, con las habilidades sociales, siempre apuntando a las posibilidades que el recurso Playcomic ofrece y que se vinculan con aspectos de la narrativa, posibilitando diferentes niveles de acceso y de abstracción.

\section{Conclusiones acerca de la experiencia}

En general, la experiencia realizada resultó interesante porque se trató de ofrecer insumos teóricos a los estudiantes, propios del campo disciplinar de Educación Especial. En este sentido, la experiencia fue productiva y desafiante. Sin embargo, también se presentaron algunas dificultades vinculadas con la complejidad en la comprensión de lo que significa el lenguaje tecnológico y el encuadre teórico que sustentamos desde nuestro proyecto de investigación, el cual fue el insumo o base de la programación de la asignatura de la cual se deriva la experiencia que narramos.

En este sentido, es muy importante volver a destacar nuestra convicción respecto a que la forma también es contenido. Para ejemplificar algunas de las dificultades que fueron apareciendo con este grupo de estudiantes, destacamos estas expresiones: «verdaderamente, la tecnología no es mi fuerte», "cuando debo aplicar lo que me piden, me pongo muy ansiosa, me nublo y siento que con la tecnología no voy a poder; no es lo mío», etc. También hubo expectativas positivas por parte de los estudiantes, traducidas en las siguientes expresiones: «me gustaría aprender cuáles son las herramientas tecnológicas con las que podemos traba- 
jar en el grado, ya que la tecnología es una metodología valiosa y rica en recursos e innovación», «me interesa aprender a utilizar herramientas tecnológicas que luego me sirvan para aplicarlas en el aula y poder, de esta forma, ofrecer distintas maneras de acceder al conocimiento», etc.

La experiencia llevada a cabo con este grupo de estudiantes nos resultó interesante, desafiante y productiva. No desconocemos la gran complejidad que supone la modificación de las matrices subjetivas de los docentes y de la comunidad educativa en general en cuanto al uso de la tecnología con vistas a procesos educativos inclusivos. En esta línea de pensamiento nos adherimos a los postulados de Booth y Ainscow (2004), para quienes la inclusión implica que los centros o las escuelas realicen un análisis crítico sobre lo que se puede hacer para mejorar el aprendizaje y la participación de todos.

Nos encontramos frente a un cambio de escenario que requiere, de parte de todo el sistema educativo, una fuerte y continua capacidad de autorreflexión, exigiendo que el sistema se adecue para atender a la diversidad en su amplio espectro y no haciendo un reduccionismo simplista, pensando que la diversidad solo tiene que ver con las personas con discapacidad
Nos adherimos a los postulados de Booth y Ainscow, para quienes la inclusión implica que los centros o las escuelas realicen un análisis crítico sobre lo que se puede hacer para mejorar el aprendizaje y la participación de todos
Consideramos que nos encontramos frente a un cambio de escenario que requiere, de parte de todo el sistema educativo, una fuerte y continua capacidad de autorreflexión, ya que los actuales lineamientos legales fomentan cada vez más el desarrollo de procesos inclusivos a nivel educativo, exigiendo que el sistema se adecue para atender a la diversidad en su amplio espectro y no haciendo un reduccionismo simplista, pensando que la diversidad solo tiene que ver con las personas con discapacidad.

\section{Consideraciones finales}

Como formadores de formadores, nos preguntamos: ¿es posible comprender estos aspectos y asumir que educar es un acto sumamente complejo, que irá abriendo los caminos necesarios para que la apropiación del lenguaje tecnológico y todas las posibilidades que ofrece se constituyan en un aliado pedagógico que colabore con entornos educativos inclusivos?

Finalmente, consideramos que debemos seguir trabajando para que en el cuadrilátero que conforma la realidad de la educación (conocimiento-docente-alumno-contexto) surjan nuevos posicionamientos en cuanto a cómo y con qué recursos enseñar, qué, para quiénes y con qué sentido. 
Debemos ser capaces de tomar la iniciativa y asumir los nuevos tiempos; salir de la zona de confort y arriesgarnos a modificar las viejas estructuras; desterrar el temor a lo desconocido y aprender alegremente junto con los estudiantes. Apropiarnos del lenguaje tecnológico es un gran paso.

\section{Referencias bibliográficas}

Booth, T. y Ainscow, M. (2004). Índice de inclusión: desarrollando el aprendizaje en la escuela. Santiago de Chile: United Nations Educational, Scientific and Cultural Organization/Oficina Regional de Educación para América Latina y el Caribe.

Facultad de Ciencias Humanas de la Universidad Nacional de San Luis. (2018). Programa 2018 de la asignatura Tecnologías Aplicadas a la Educación Inclusiva. Ciclo Complementario de Licenciatura en Educación Especial.

Muñoz Germán, A. (2009). Guía detallada de Playcomic. Recuperado de <http://ntic.edu cacion.es/w3/eos/MaterialesEducativos/ mem2009/playcomic/index_es.html> (consultado en julio de 2019).

Pahud, F. y Zuñiga, M. (2018). El enriquecimiento del lenguaje tecnológico en la formación del profesor de educación especial. Eje temático II: La formación del profesor y licenciado en educación especial. En M. ${ }^{2}$ F. Pahud y C. M. Cavallero (Comp.), Libro de resúmenes de trabajos presentados: Rupturas y continui-

dades de la educación especial hoy/Debates pendientes hacia la educación inclusiva, XXVI Jornadas Nacionales de la Red Universitaria de Educación Especial (RUEDES) y XX Jornadas Nacionales de la Red de Estudiantes de Carreras y Cátedras de Educación Especial (RECCEE), celebradas el 5, 6 y 7 de octubre de 2017, Facultad de Ciencias Humanas, Universidad de San Luis. San Luis, Argentina: Nueva Editorial Universitaria. Recuperado de <http://www.neu.unsl.edu. ar/wp-content/uploads/2018/03/Libro-deresu\%CC\%81menes-RUEDES_2017.pdf> (consultado en julio de 2019).

Villagrán, S. (2018). Estrategias inclusivas en la escuela: la planificación multinivel. Documento de cátedra. Ciclo Complementario de Licenciatura en Educación Especial. Facultad de Ciencias Humanas de la Universidad Nacional de San Luis.

Zuñiga, M. y Pahud, F. (2016). El lenguaje tecnológico en las prácticas de enseñanza en la formación del profesorado. Argonautas, 6(7), 134-143. 